\title{
1 The methyl cycle is a conserved regulator of biological clocks
}

2

3 Jean-Michel Fustin ${ }^{1 *}$, Shiqi Ye ${ }^{1 \dagger}$, Christin Rakers ${ }^{2}$, Marijke Versteven ${ }^{3}$, Samantha J.

4 Cargill $^{4}$, T. Katherine Tamai ${ }^{5}$, Yao Xu ${ }^{6}$, Maria Luísa Jabbur ${ }^{6}$, Rika Kojima ${ }^{7}$, Melisa

5 L. Lamberti ${ }^{8}$, Kumiko Yoshioka-Kobayashi ${ }^{9}$, David Whitmore ${ }^{10}$, Ryoichiro

6 Kageyama $^{9}$, Takuya Matsuo ${ }^{11}$, Ralf Stanewsky ${ }^{3}$, Diego A. Golombek ${ }^{8}$, Carl Hirschie

7 Johnson $^{6}$, Gerben van Ooijen ${ }^{4}$, Hitoshi Okamura ${ }^{12 *}$.

8

91 Graduate School of Pharmaceutical Sciences, Laboratory of Molecular Metabology,

10 Kyoto University, Japan. Tel: +81-75-753-9254

112 Graduate School of Pharmaceutical Sciences, Kyoto University, Japan

123 Institute of Neuro- and Behavioral Biology, University of Münster,

13 Münster

144 School of Biological Sciences, University of Edinburgh, United Kingdom

155 Institute of Transformative Bio-Molecules (WPI-ITbM), Nagoya University,

16 Nagoya, Japan

176 Department of Biological Sciences, Vanderbilt University, Nashville, USA

187 Karolinska Institute, Sweden

198 Department of Science and Technology, National University of

20 Quilmes/CONICET, Buenos Aires, Argentina

219 Institute for Frontier Life and Medical Sciences, Kyoto University, Kyoto, Japan

2210 Centre for Cell and Molecular Dynamics, Department of Cell and Developmental

23 Biology, University College London, United Kingdom

2411 Center for Gene Research, Nagoya University, Nagoya, Japan 
2512 Graduate School of Pharmaceutical Sciences, Molecular Brain Science, Kyoto

26 University, Kyoto, Japan.

$28+$ These authors contributed equally to this work

$29 *$ Corresponding authors: j.m.fustin@pharm.kyoto-u.ac.jp and

30 okamurah@pharm.kyoto-u.ac.jp

\section{Abstract}

The methyl cycle is a universally conserved metabolic pathway operating in prokaryotes and eukaryotes. In this pathway, the amino acid methionine is used to synthesize S-adenosylmethionine, the methyl donor co-substrate in the methylation of nucleic acids, histone and non-histone proteins and many other molecules within the cell. The methylation of nucleic acids and proteins is the foundation of epigenetic and epitranscriptomic regulations of gene expression, but whether the methyl cycle centrally regulates gene expression and function by controlling the availability of methyl moieties is poorly understood.

42 From cyanobacteria to humans, a circadian clock that involves an exquisitely regulated transcription-translation-feedback loop driving oscillations in gene expression and orchestrating physiology and behavior has been described. We reported previously that inhibition of the methyl cycle in mammalian cells caused the lengthening of the period of these oscillations, suggesting the methyl cycle may

47 indeed act as a central regulator of gene expression, at least in mammals. Here, we 48 investigated whether the methyl cycle, given its universal presence among living 49 beings, regulates the circadian clock in species across the phylogenetic tree of life. 
50 We reveal a remarkable evolutionary conservation of the link between the methyl

51 cycle and the circadian clock. Moreover, we show that the methyl cycle also regulates

52 the somite segmentation clock, another transcription-translation negative feedback

53 loop-based timing mechanism that orchestrate embryonic development in vertebrates,

54 highlighting the methyl cycle as a master regulator of biological clocks.

55

SIGNIFICANCE STATEMENT

Here we reveal that the methyl cycle, a universal metabolic pathway leading to the synthesis of S-adenosylmethionine, the methyl donor co-substrate in virtually all transmethylation reactions within the cell, is a conserved regulator of biological clocks. These discoveries highlight the methyl cycle as a metabolic hub that regulates gene expression via the availability of methyl moieties for the methylation of nucleic acids, proteins and many other molecules with the cell.

\section{INTRODUCTION}

Methylation reactions start with the metabolization of methionine into $S$ adenosylmethionine, or SAM: the universal methyl donor co-substrate in the transmethylation of nucleic acids, proteins, carbohydrates, phospholipids and small molecules. During the methylation process, SAM is converted into adenosylhomocysteine (SAH) that is rapidly hydrolysed into homocysteine to prevent competitive inhibition of methyltransferase enzymes by SAH due to its close structural relatedness to SAM. Homocysteine is detrimental to physiology (1), and is recycled back into methionine or used for glutathione synthesis via cysteine and cystathionine in the trans-sulfuration pathway. The ratio SAM/SAH is known as the methylation potential: a measure of the tendency to methylate biomolecules (2-4). In 
plants, fungi, prokaryotes and other microorganisms, the methyl cycle is used both as a source of SAM, as well as a source of de novo methionine synthesis for growth. In a wide variety of eukaryotes, however, methionine is an essential amino acid that must be obtained from food, especially in periods of growth, the methyl cycle in these organisms ensuring only the flow of one-carbon units to SAM.

80 Three enzymatic activities are required to keep the methyl cycle running. 5-methyltetrahydrofolate or betaine are used as a source of methyl moieties to regenerate methionine (Met) from homocysteine (Hcy). The enzyme Methyltetrahydrofolatehomocysteine S-methyltransferase is universal but Betaine-homocysteine methyltransferase is present only in some vertebrate tissues, mainly in the liver. Likewise, Adenosylmethionine synthetase (SAM synthesis from Met) and adenosylhomocysteinase (Hcy synthesis from SAH) are virtually universal. There is considerable divergence in methyltransferases (MTases), and the phylogeny of five structural classes of MTases is unclear. The oldest MTases represents the RNA methyltransferases, appearing in the early forms of life evolving on Earth in an RNA World (5). Methyl cycle metabolites are thought to have been present in the prebiotic world, since methionine can be created by a spark discharge and is proposed to be an intermediate in the prebiotic synthesis of homocysteine, which was also found among organic molecules synthesized in the 1972 Miller experiment (6). Prebiotic chemistry might have involved methylation by reaction with formaldehyde, an abundant prebiotic organic molecule, to prime the evolution of biological methylation reactions (7).

98 Similarly, an endogenous circadian clock evolved to anticipate the daily cycles of 99 light and darkness has been found in many organisms, from cyanobacteria to humans. 
100 Transcription-translation feedback loops (TTFLs) of "clock genes" directly or

101 indirectly regulating their own transcription underlie many functions of the clock, and

102 drive oscillations of output genes controlling physiology and behavior. Some

103 molecular components of the clock are remarkably conserved in metazoan, notably

104 the genes Clock and Period, coding for transcription factors, with Clock activating the

105 transcription of Per and Per inhibiting its own transcription.

107 In 2013, we reported that disruption of the methyl cycle by inhibitors of 108 Adenosylhomocysteinase (AHCY), causing an accumulation of SAH known to lead to 109 a general inhibition of transmethylations, strongly affected the circadian clock in

110 mouse and human cells (8). We now show that the link between the methyl cycle and

111 the circadian clock we first uncovered in mammals has been conserved during more

112 than 2.5 billion years of evolution. We further reveal a similar link between the 113 methyl cycle and another TTFL called the somite segmentation clock that underlies 114 the development of early body plan in vertebrates, indicating that the methyl cycle is a 115 key regulator of biological clocks.

117 RESULTS

118 AHCY is a remarkably conserved key enzyme in the methyl cycle

119 The use of carbocyclic adenosine analogues such as Deazaneplanocin A (DZnep) as 120 inhibitors of AHCY was established more than 30 years ago (9-11). The reaction 121 catalyzed by AHCY is the cleavage of SAH to adenosine and L-homocysteine, DZnep 122 inhibiting this reaction by occupying the adenosine binding site. The crystal structure 123 of human (14), mouse (15) and yellow lupin (Lupinus luteus) (16) AHCY 124 complexed with adenosine or analogues have been described, and insights into its 
125 catalytic activity have been obtained (17-20). AHCY has been reported to be one of

126 the most evolutionarily conserved proteins (21), but experimentally-determined

127 structures of AHCY with DZnep remain to be described for most organisms

128 investigated here. A full-length multiple sequence alignment (Fig. S1) and homology

129 modelling of AHCY from human to cyanobacteria (Fig. 1a and Movie S1) revealed

130 high sequence and predicted tertiary structure conservation. Amino acids contributing

131 to the DZnep binding site showed at least $88 \%$ identity between all eukaryotic AHCY

132 sequences, and $78 \%$ between human and bacterial sequences, resulting in the DZnep

133 binding site to be virtually identical in all organisms investigated (Fig. 1b, c and Fig.

134 S2). Moreover, amino acids that were reported as crucial for the activity of rat AHCY

135 (His55, Asp130, Glu155, Lys186, Asp190, and Asn191 (20)) are perfectly conserved

136 (Fig. S1). In line with the above, molecular docking simulations of AHCY with

137 adenosine or DZnep revealed comparable ligand binding conformations and estimated

138 binding free energies for all organisms (Fig. 1d and S2). Together these observations

139 strongly support the use of DZnep as a valid approach to test the effects of methyl

140 cycle inhibition on clocks across phyla.

142 The circadian clock and methyl cycle are linked in vertebrates.

143 We first tested the effects of DZnep on the mammalian clock, in a human

144 osteosarcoma cell line stably transfected with a luciferase reporter vector for the core

145 clock gene Bmal1 (22) and in mouse embryonic fibroblasts from PER2::LUC knock-

146 in mice expressing a fusion between the endogenous core clock protein PER2 and

147 LUCIFERASE (23). These cells are the gold standard for measuring circadian

148 parameters in vitro since they allow the oscillating expression of the genes Bmall or

149 Per2 to be followed in real-time. In human (Fig. 2a) and mouse (Fig. 2b) cells, DZnep 
150 dose-dependently increased the period, up to a maximum of $\sim 40$ hours and $\sim 30$ hours,

151 respectively, with $100 \mu \mathrm{M}$ DZnep.

152 We next tested DZnep on a non-mammalian cell type commonly used for circadian

153 studies, the zebrafish embryonic PAC2 cell line and revealed a potent effect of the

154 drug both on circadian period and amplitude (Fig. 2c), as in mammals. Since the

155 circadian clock in these cells is directly light-sensitive and can be entrained by light-

156 dark cycles, we also tested whether the entrainment of the cells to light was affected

157 by DZnep before release into constant darkness (DD). Indeed, a strong effect on

158 entrainment was observed (Fig. 2c). The gene Perlb normally peaks at dawn but was

159 severely blunted and delayed in DZNep-treated cells, even at the lowest concentration

160 of DZnep. In DD, period lengthened dramatically to a peak value of over 40 hours at

$16110 \mu \mathrm{M}$, that was slightly lower at $100 \mu \mathrm{M}$. To conclude, period and amplitude of

162 circadian oscillations of clock gene expression are strongly affected by DZnep in all 163 three vertebrate cell systems.

165 The circadian clock and methyl cycle are linked in invertebrates.

166 As models for invertebrates, we selected the fruit fly Drosophila melanogaster and

167 the nematode Caenorhabditis elegans. Flies have been instrumental in molecular 168 circadian biology, allowing the 2017 Nobel laureates and their co-workers to establish

169 the canonical TTFL model underlying circadian oscillations in metazoan (24-26).

170 In contrast, C. elegans is a relatively new model in circadian biology, the general 171 principles governing its circadian clock remaining largely unidentified (27, 28).

172 Circadian oscillations in the expression of the sur-5 gene were described a few years 173 ago and used for the real-time monitoring of the nematode's circadian molecular 174 rhythms by luciferase reporter (29). 
175 As observed in vertebrates, DZnep caused dose-dependent period lengthening in $D$. melanogaster haltere cultures from transgenic luciferase reporter TIM-LUC flies (Fig.

$1773 \mathrm{a}$ ), and a significant effect of $100 \mu \mathrm{M}$ DZnep was also observed in freely moving 178 nematodes. Period changes in flies were of lesser magnitude than observed in 179 vertebrates. Nevertheless, these results indicate that the link between the circadian 180 clock and the methyl cycle is conserved in invertebrates.

\section{The circadian clock and methyl cycle are linked in plants and algae.}

183 Fig. 1 and S1 show that a plant- and green algae-specific region exists in AHCY, from amino acids 151 to 191 , which is involved in the interaction of AHCY with adenosine kinase and cap methyltransferase, and required for nuclear targeting of the enzyme $(30,31)$. Despite this insertion, however, the domains for $\mathrm{SAH}$ and $\mathrm{NAD}^{+}$binding are remarkably conserved.

188 The most commonly used model organism to study circadian rhythms in land plants is 189 Arabidopsis thaliana. We thus tested the effects of DZnep on luminescent rhythms reporting the expression of the plant evening gene TOC1 in protoplasts (Fig. 4a), and extended our investigations to aquatic unicellular green algae (Fig. $4 \mathrm{~b}$ and c).

192 Ostreococcus tauri and Chlamydomonas reinhardtii represent two different classes of

193 unicellular green algae that have been successfully used in circadian studies and 194 constitute great models to investigate cell-autonomous metabolic processes (32-36).

195 We tested increasing concentration of DZnep on Arabidopsis and algal cell types and 196 observed an increase in period length and a decrease in amplitude almost identical to 197 the results obtained in vertebrates. In conclusion, the effects of DZnep treatment on 198 transcriptional rhythms are also conserved in the plant kingdom. These results are 
especially significant given unicellular algae and humans are separated by more than

2001 billion years of evolution.

The circadian clock and methyl cycle are linked in prokaryotes.

203 Eukaryotic circadian clocks involve a complex TTFL system that requires regulated 204 gene expression at every level from transcriptional to post-translational steps: DNA methylation and the histone code, RNA processing, translation efficiency, 206 phosphorylation and other protein modifications (37). In contrast, a biochemical 207 oscillator in the cyanobacteria Synechococcus elongatus can independently generate a 208 24-hour rhythm. Three proteins, Kai-A, -B and -C, form a nanocomplex that 209 regulates two key activities of KaiC: ATPase and autophosphorylation. The result is 210 an autonomous and self-sustained phosphorylation-based oscillator ticking with a

211 period close to 24-hours. In a cellular context, this non-transcriptional oscillator 212 controls transcriptional outputs that in turn add robustness to the biochemical 213 oscillator via TTFLs (38-42).

214 Like all organisms, cyanobacteria synthesize SAM, required for essential 215 transmethylations. In cyanobacteria we observed a more complex response to DZnep 216 than in other organisms, perhaps due to the presence in these cells of a self-sustained 217 biochemical oscillator that drives the TTFL system. While circadian period 218 lengthened in response to low concentrations of DZnep, the amplitude increased (Fig. 219 5a). These observations were confirmed using a different reporter strain of $S$. 220 elongatus (Fig. 5b). Probing the methyl cycle-clock relationship further revealed that 221 intermediate concentrations of the drug caused no significant effect on period but still 222 significantly increased the amplitude (Fig. S3). At $200 \mu \mathrm{M}$ however, a dramatic 
223 reverse effect on oscillations was seen, the amplitude almost completely collapsing

224 (Fig. S3).

225 Inhibition of methyltransferases by DZnep depends on the unique activity of AHCY

226 to hydrolyze SAH. Some bacterial proteins, such as Mtn in E.coli or Pfu in

227 Streptococcus pyogenes, however possess a SAH nucleosidase activity that cleaves

228 SAH to adenine and ribosylhomocysteine (43). If this pathway for AHCY catabolism

229 is active in Synechococcus elongatus, acting as a buffer against SAH accumulation, it

230 would explain the blunted period response to DZnep compared to eukaryotes. Indeed,

231 SAH nucleosidases have been identified in at least some Synechococcus strains, such

232 as PCC7336 and MED-G69 (44). To circumvent the potential activity of a SAH

233 nucleosidase in Synechococcus elongatus, we decided to use the global methylation

234 inhibitor sinefungin, a natural analogue of SAM that directly binds to and inhibit

235 methyltransferases (45, 46). A known antifungal and antibacterial agent whose

236 activity as such has been shown to depend at least partially on the inhibition of

237 mRNA 5'-cap methylation (47), it was previously used at 10 and $100 \mu \mathrm{M}$ on the

238 cyanobacteria Anabaena to elicit non-lethal methylation-dependent morphological

239 changes (48). We thus selected Sinefungin to further probe the link between

240 methyltransferases and the circadian period in Synechococcus elongatus. In both

241 reporter strains, significant dose-dependent period lengthening was observed. To

242 contrast with Sinefungin, we also tested a selective bacterial DNA methyltransferase

243 inhibitor with a different molecular footprint from DZnep or Sinefungin, the

244 cyclopentaquinoline carboxylic acid EGX ${ }^{\mathrm{I}}$ (8-ethoxy-6-nitro-3a,4,5,9b-tetrahydro-3H-

245 cyclopenta[c]quinoline-4-carboxylic acid) (49) (Fig. S4). Since its published IC 50 for

246 E. coli DNA methyltransferase is $9.7 \mu \mathrm{M}$ (49), we used EGX ${ }^{\mathrm{I}}$ at 5 and $50 \mu \mathrm{M}$ but did

247 not observe any consistent effects on the period, with only a mild period shortening in 
249 some growth inhibition during the experiment, but Synechococcus still showed

250 healthy rhythms. In contrast, at $100 \mu \mathrm{M}$ or higher $\mathrm{EGX}^{\mathrm{I}}$ was very toxic to

251 cyanobacteria, causing a dramatic collapse of luciferase intensity, with poor rhythms

252 from which circadian parameters could not be reliably extracted.

253 Together these data show that the circadian clock in cyanobacteria - separated by 2

254 billion years of evolution from humans - is sensitive to methylation inhibition. This

255 is especially meaningful considering that the cyanobacterial core clock is a

256 phosphorylation-based biochemical oscillator. This also underlines the fundamental

257 role that methyl metabolism kept in the control of physiology and behavior across

258 evolution. Alternative metabolic pathways branching from the methyl cycle may

259 prevent the accumulation of SAH and make prokaryotes more resistant to methylation

260 inhibition by nutrient deprivation.

\section{The somite segmentation clock and methyl cycle are linked}

263 Our results so far have shown that the link between the methyl cycle and the circadian

264 TTFL is conserved. What about other biological timekeepers? While the circadian 265 clock involves a TTFL that oscillates with a period near 24 hours, another TTFL, 266 called the somite segmentation clock, cycles much faster and orchestrates the 267 appearance of new somites from the paraxial mesoderm of the developing embryo in 268 vertebrates (Fig. 6). In mouse, the underlying molecular oscillator is centered on the 269 transcription factor Hairy \& Enhancer Of Split 7 (Hes7), whose expression oscillates 270 with a period close to 2 hours by negative feedback that also induces oscillations of $271 \quad$ Notch and Fgf signaling $(50,51)$. 
272 Like circadian clock genes, Hes 7 oscillatory expression can be monitored in real-time

273 from transgenic embryo expressing highly destabilized luciferase under the control of

274 the Hes7 promoter. Testing increasing concentrations of DZnep on these transgenic

275 embryos revealed uncanny similarities with results seen so far: an increase in period

276 length accompanied by a decrease in amplitude of Hes 7 oscillations (Fig. 6). This

277 once more illustrates the importance of the methyl cycle in the regulation of

278 transcriptional programs orchestrating development, physiology and behaviour.

280 Here we provide evidence for the existence of an ancient link between the methyl

281 cycle and biological timekeepers. In the course of more than 2 billion years of cellular

282 evolution, during which the biological clock and the methyl cycle have adapted to a

283 multicellular eukaryotic existence, the link between these two processes has remained

284 active. Further investigations should reveal how the link itself has evolved, and which

285 methylated substrates and methyltransferases are critical for its function.

\section{DISCUSSION}

289 Solar ultraviolet radiation played a critical role in prebiotic chemistry and has been

290 involved in the origin of chirality (52), the synthesis of alcohols, aldehydes (notably

291 formaldehyde), and organic acids (53), as well as that of amino acids (54) and

292 ribonucleotides (55). The presence of methylation transfer in the prebiotic world 293 probably caused the incorporation of methyl chemistry in early life-forms. The energy

294 of UV photons however can also degrade biologically important molecules, 295 preventing abiogenesis. Once autocatalytic pseudo-life forms evolved with more 296 complex chemistry, avoidance mechanisms may have provided increased fitness in 
such an environment. The origin of the circadian clock on the early Earth is likely to have been a simple sensing of molecules present in the milieu, increasing or

299 decreasing under UV exposure and triggering an appropriate response. Formaldehyde, 300 increasing during the day under the action of UV on carbon monoxide and water 301 vapor (53), could have been such a chemical marker of daylight, affecting the 302 chemistry of early life-forms by methylation. A conserved link between methyl transfer and the circadian clock may have arisen from such a scenario.

The most surprising data obtained here is the almost identical response of unicellular 306 algae and vertebrates to DZnep despite their evolutionary divergence more than 1 307 billion years ago, the comparable effect of methylation inhibitors on cyanobacterial 308 circadian clock ( 2 billion years of evolutionary divergence), and the conservation of 309 this biochemical link in the control of the somite segmentation clock. So far, only a 310 small number of examples have been reported that identified aspects of circadian

311 timekeeping shared among rhythmic life, such as oxidation cycles of peroxiredoxin $312(34,56,57)$ and regulation by oscillating intracellular magnesium concentrations 313 (58).

315 In mammalian cells we previously reported that methyl cycle inhibition decreased the 316 methylation of internal adenosines in mRNA (m6A), as well as that of histones (8). 317 While specific m6A inhibition was sufficient to elicit period elongation, the 318 contribution of histone methylation to the period elongation obtained by DZnep was 319 likely significant, as well as that of other methylation sites in mRNA, rRNA and 320 tRNA. Due to the considerable heterogeneity in mechanisms regulating gene 321 expression and function in organisms tested here, identifying a single mechanism 
explaining period elongation would be a difficult undertaking and is beyond the scope

323 of the present work. We mentioned in the introduction that the oldest MTases are RNA methyltransferases (5), it is therefore tempting to propose that inhibition of RNA methylation may at least partially contribute to the period lengthening. That sinefungin, a confirmed mRNA cap-methyltransferase inhibitor (47), was able to

327 lengthen the cyanobacterial clock period while EGX, a DNA methylation inhibitor, was not support this hypothesis. It is also possible that the effects of methyl cycle inhibition on period and amplitude in eukaryotes arise from different mechanisms, e. g. on the inhibition of mRNA and histone methylation, respectively. More 331 experiments should clarify these points.

The presence of a SAH nucleosidase in S. elongatus may have blunted the effect of DZnep, but even with $100 \mu \mathrm{M}$ sinefungin the period lengthened only about 2 hours. It

335 should be mentioned that our luciferase reporter system is a reporter for the driven

336 TTFL, not for the KaiABC oscillator per se. It is therefore possible that methylation 337 inhibition only affected the coupling between the nanocomplex and the TTFL, which 338 may explain why the increase in period was less pronounced compared to most 339 eukaryotes tested here. In eukaryotic cells, luciferase reporter systems are a direct 340 read-out of the core oscillator that has evolved more dependent on the TTFL, and as a 341 result may have become more sensitive to perturbations affecting gene expression 342 such as inhibition of RNA and histone methylation.

344 The somite segmentation clock was strongly affected by inhibition of AHCY, which 345 is in line with the importance of 1 carbon metabolism for embryonic development. As 346 can be seen in the Movie S3, showing similar results to Movie S2 but merged with 
347 brightfield images and displaying luminescence as a pseudo-color green, period

348 lengthening of the oscillatory expression of Hes7-luciferase occurred together with a

349 pronounced delay in the growth of the caudal tip of the presomitic mesoderm as well

350 as in the appearance of new somites, demonstrating that the molecular clock as well

351 as its output, i.e. the somitogenesis, were affected by DZnep. The mechanisms

352 underlying this period lengthening may be distinct from those involved in the

353 lengthening of the circadian period. Considering the short period of Hes 7 oscillations

$354-2 \mathrm{~h}$, and the importance of 3'-UTR-dependent regulation of Hes $7 \mathrm{mRNA}$ turnover

355 for its cyclic expression (62), the inhibition of mRNA methylation may at least in

356 part contribute to the results observed.

358 Despite being one of the most potent AHCY inhibitors (11), DZnep is sometimes

359 erroneously sold and used as a "specific" histone methyltransferase EZH2 inhibitor

360 because of the misinterpretation of a report showing that it inhibits - without any data

361 on specificity - histone methylation by EZH2 in cancer cells (59). More recent

362 reports showed that, in line with its inhibitory effect on AHCY $(60,61)$, DZnep

363 globally inhibits histone methylation.

365 Since we sought to inhibit the methyl cycle in organisms from bacteria to humans,

366 these investigations were by necessity limited to the pharmacological inhibition of

367 AHCY, because irreversible genetic disruption of AHCY in all organisms would have 368 been much less feasible, notably due to likely embryonic lethality or developmental 369 arrest in metazoa, cell cycle/growth disruption, and the existence of multiple 370 uncharacterized homologues in many organisms tested here. This work puts the 
371 spotlight on the methyl cycle and calls for more investigations into how it regulates

372 physiology and behavior.

\section{AKNOWLEDGEMENTS}

375 This work was supported in part by the Ministry of Education, Culture, Sports, 376 Science and Technology of Japan: Grant-in-aid for Scientific Research on Innovative 377 Areas (26116713, J.-M. F.), for Young Scientists (26870283, J.-M. F.), for Scientific 378 Research A (15H01843, H.O.), a grant for Core Research for Evolutional Science and 379 Technology, Japan Science and Technology Agency (CREST/JPMJCR14W3, H.O.). 380 J.-M.F. was also supported by grants from the Kato Memorial Bioscience Foundation, 381 the Senri Life Science Foundation (S-26003), the Mochida Memorial Foundation for 382 Medical and Pharmaceutical Research, and the Kyoto University internal grant 383 ISHIZUE, and H.O. was also supported by the Kobayashi International Scholarship 384 Foundation. C.H.J. is supported by grants from the USA NIH/NIGMS: GM067152 385 and GM107434. G.vO. is supported by a Royal Society University 386 Research Fellowship (UF160685) and research grant (RGF\EA \180192). D.A.G. is 387 supported by grants from the National Research Agency (ANPCyT, PICT-2015-0572) 388 and the National University of Quilmes. We thank Adrienne K. Mehalow, Jay C. 389 Dunlap, John O'Neill and Tokitaka Oyama for useful discussion and for kindly 390 accepting to perform experiments. The authors thank G. Wolber, Freie Universität 391 Berlin, Germany, for providing a LigandScout 4.2 license.

\section{Author Contributions}

394 Conceptualization, J.-M.F. and H.O.; Methodology, J.-M.F., C.R., M.V., S.J.C., 395 T.K.T., Y.X., M.L.J., M.L.L., K.Y.-K., D.W., R.Ka., T.M., R.S., D.A.G., C.H.J., 
397 M.L.L., K.Y.-K., T.M., D.A.G., G.v.O.; homology modelling, C.R.; original draft, J.-

398 M.F.; reviewing \& editing, J.-M.F., C.R., T.K.T, Y.X., K.Y.-K., D.W., R.Ka., T.M.,

399 R.S., D.A.G., C.H.J., G.v.O.; Funding Acquisition, J.-M.F., D.A.G., C.H.J., G.v.O

400 and H.O.; Supervision, J.-M.F.

401

402 
METHODS

\section{Molecular modelling and sequence analyses}

406 Homology models were obtained as described in the SI Appendix.

Assay for effect of the inhibition of the methyl cycle on vertebrate circadian clock.

411 and mouse PER2::LUC MEFs (23) cell lines were cultivated as previously described

412 (8). Briefly, cells were seeded into $35 \mathrm{~mm}$ dishes (Corning) and allow to grow to 413 confluence in DMEM/F12 medium (Nacalai) containing 414 penicillin/streptomycine/amphotericin (Nacalai). Cells were then shocked by 415 dexamethasone (Sigma-Aldrich) $200 \mathrm{nM}$ for 2 hours, followed by a medium changed 416 including $1 \mathrm{mM}$ luciferine (Nacalai). $35 \mathrm{~mm}$ dishes were then transferred to an 8 417 dishes luminometer-incubator (Kronos Dio, Atto). Photons were counted in bins of 2 $418 \mathrm{~min}$ at a frequency of $10 \mathrm{~min}$. DZnep was purchased from Sigma-Aldrich.

419 Zebrafish cell line and bioluminescence assays. The generation of a perlb-luciferase 420 cell line from zebrafish PAC2 cells has been previously described (64). Cells were 421 cultured in Leibovitz's L-15 medium (Gibco) containing 15\% fetal bovine serum 422 (Biochrom AG), $50 \mathrm{U} / \mathrm{mL}$ penicillin/streptomycin (Gibco), and $50 \mu \mathrm{g} / \mathrm{mL}$ gentamicin 423 (Gibco). Cells were seeded at a density of 50,000-100,000 cells per well in 424 quadruplicate wells of a 96-well plate in medium supplemented with $0.5 \mathrm{mM}$ beetle 425 luciferin (Promega), and drugs were prepared in water and added at the concentrations 
426 indicated in the figures and legends. Plates were sealed with clear adhesive TopSeal

427 (Perkin Elmer, Waltham, MA, USA). Cells were exposed to a 12:12 LD cycle for 7

428 days and then transferred into DD for at least 3 days. Bioluminescence was

429 monitored on a Packard TopCount NXT scintillation counter $\left(28^{\circ} \mathrm{C}\right)$. DZnep was

430 purchased from Sigma-Aldrich.

431 Period and amplitude were estimated by BioDare2 (63).

\section{Assay for effect of the inhibition of the methyl cycle on invertebrate circadian}

433 clock.

434 Halteres of two- to seven-day old transgenic ptim-TIM-LUC males (65) kept under 12

$435 \mathrm{~h}: 12 \mathrm{~h}$ light:dark cycles (LD) at $25^{\circ} \mathrm{C}$ were bilaterally dry dissected. Each pair was

436 transferred into one well of a 96 well plate (Topcount, Perkin Elmer) filled with

437 medium containing 80\% Schneider's medium (Sigma), 20\% inactivated Fetal Bovine

438 Serum (Capricorn) and 1\% PenStrep (Sigma). Medium was fortified with $226 \mu \mathrm{M}$

439 Luciferin (Biosynth) and supplemented with 10, 100, or $250 \mu \mathrm{M}$ Dznep A diluted in

440 PBS. Plates were sealed with clear adhesive covers and transferred to a TopCount

441 plate reader (PerkinElmer). Bioluminescence emanating from each well was

442 measured hourly in LD for two days, followed by 5 days of constant darkness (DD) at

$44325^{\circ} \mathrm{C}$ as previously described (66). The ptim-TIM-LUC reporter contains the timeless

444 (tim) promoter sequences driving rhythmic expression of the tim cDNA, which is

445 fused to the firefly luciferase cDNA.

446 C. elegans strain N2 (Bristol strain, wild-type was provided by the Caenorhabditis

447 Genetics Center, University of Minnesota (cbs.umn.edu/cgc/home). Stocks were

448 maintained on plates with nematode growth medium (NGM) seeded with 
449 HB101 Escherichia coli strain, under 12-h/12-h LD/CW cycle (400/0 1x and CW

$450\left(18.5 / 20{ }^{\circ} \mathrm{C}, \Delta=1.5 \pm 0.125{ }^{\circ} \mathrm{C}\right)$ environmental cycles. Transgenic animals were

451 generated by microinjection of a Psur-5::luc::gfp construct at 50 or $100 \mathrm{ng} / \mu \mathrm{L}$ with 452 the pRF4 marker $(100 \mathrm{ng} / \mu \mathrm{L}) \quad(29,67)$. Bioluminescence recordings with nematodes 453 are described in the SI Appendix.

454 Period and amplitude were estimated by BioDare2 (63). clock.

Ostreococcus tauri cells transgenically expressing a translational fusion of CCA1 to luciferase from the CCA1 promoter (CCA1-LUC) (36) were grown, imaged, and analysed as described previously (58).

For protoplast isolation and luminescent imaging, plants expressing luciferase from

461 the CCA1 promoter in the Col-0 background (kindly provided by Karen Halliday, 462 University of Edinburgh) were grown in sterile soil under long-day conditions (16 h 463 light $/ 8 \mathrm{~h}$ dark) at $22^{\circ} \mathrm{C}$ under $60-70 \mu \mathrm{mol} \mathrm{m} \mathrm{m}^{-2} \mathrm{~s}^{-1}$ white LED tube lights (Impact T8).

464 Protoplasts were isolated from 3-week old leaves as previously described (68). 465 Protoplasts in a solution of $1 \mathrm{mM}$ D-luciferin (Biosynth AG), $5 \%$ fetal bovine serum 466 (Sigma), 50 $\mathrm{g} / \mathrm{ml}$ ampicillin, $140 \mathrm{mM} \mathrm{NaCl}, 115 \mathrm{mM} \mathrm{CaCl} 2,4.6 \mathrm{mM} \mathrm{KCl}, 1.86 \mathrm{mM}$ 467 MES pH 5.7 and $4.6 \mathrm{mM}$ glucose were added to white, flat-bottomed 96-well plates 468 (Lumitrac, Greiner Bio-one) at a concentration of $2 \times 10^{5}$ cells $/ \mathrm{ml}$. DZnep (S7120 469 Selleckchem) was added to the protoplasts to achieve concentrations of $0,0.125,0.25$, 4700.5 or $1 \mu \mathrm{M}$ from a $1 \mathrm{mM}$ stock solution (prepared in distilled $\mathrm{H}_{2} \mathrm{O}$ ) to a total volume 471 of $200 \mu \mathrm{L}$ per well. Plates were sealed with a clear adhesive lid (TopSeal-A, Perkin 
472 Elmer). Protoplast luminescence was read by a LB942 Tristar $^{2}$ plate reader (Berthold

473 Technologies Ltd) every 50 minutes for 3 seconds per well, and kept under

474 continuous red $(630 \mathrm{~nm})$ and blue $(470 \mathrm{~nm})$ LED light $\left(5 \mu \mathrm{mol} \mathrm{m} \mathrm{s}^{-2} \mathrm{~s}^{-1}\right.$ each $)$ at $19^{\circ} \mathrm{C}$.

476 Chlamydomonas reinhardtii strain CBR carrying a codon-adapted luciferase reporter

477 driven by the tufA promoter in the chloroplast genome $(69,70)$ was used. Culture 478 preparation, bioluminescence monitoring, and data analysis were carried out as 479 described previously (70). Briefly, 5-day-old algal cultures on HS agar medium were 480 cut out along with the agar by using a glass tube, and transferred to separate wells of a 481 96-well microtiter plates. Luciferin (final conc. $200 \mu \mathrm{M}$ ) and various concentrations 482 of DZnep (Sigma) were added to the wells. Algae were synchronized by a single 483 cycle of 12-h darkness/12-h light $\left(30 \mu \mathrm{mol} \mathrm{m}{ }^{-2} \mathrm{~s}^{-1}\right)$ at $17^{\circ} \mathrm{C}$ before bioluminescence 484 monitoring using a custom-made luminometer-incubator (71) in DD at $17^{\circ} \mathrm{C}$.

486 Period and amplitude were estimated by BioDare2 (63).

Assay for effect of the inhibition of the methyl cycle on prokaryotic circadian clock.

Vibrio harveyi luciferase encoded by $\operatorname{lu} x A$ and $\operatorname{lu} x B(\operatorname{lu} x A B)$ genes was used as a 490 luminescence reporter in cyanobacterium Synechococcus elongatus PCC 7942. Two 491 cyanobacterial clock-regulated reporter strains, kaiBCp::luxAB (72) and 492 psbAIp::luxAB (73) were selected for the test, i.e. expression of the lux $A B$ was under 493 control of the promoters of the central clock genes kaiBC and the Class I 494 photosynthetic gene psbAI, respectively. Synechococcus strains were grown in 495 modified BG11 media (74) supplemented with $40 \mu \mathrm{g} / \mathrm{ml}$ of spectinomycin. The 
496 cultures grown on BG11 agar plates for 3 days at $30^{\circ} \mathrm{C}$ under continuous cool-white

497 illumination (LL) $\left(50 \mu \mathrm{E} / \mathrm{m}^{2} \mathrm{~s}\right)$ were toothpicked onto fresh BG11 agar plates

498 containing $0.015 \mathrm{~g} / \mathrm{L}$ of L-methionine and different concentrations of 3-

499 deazaneplanocin A hydrochloride (Sigma), sinefungin (Abcam) or EGX

500 (ChemBridge 5790780). After a single 12h dark pulse for synchronization, assay of

501 the in vivo luminescence rhythms was performed as described previously (72).

502 Period and amplitude were estimated by BioDare2 (63).

Measurement of $\mathrm{Hes} 7$ oscillations in the mouse presomitic mesoderm

505 Presomitic mesoderm (PSM) tissues from three littermate pHes7-UbLuc Tg embryos

506 were embedded in $0.35 \%$ LMP-agarose/culture medium (10\% FBS-DMEM/F12), in a

507 silicon mold mounted onto a $\varphi 35 \mathrm{~mm}$-glass bottom dish, and luciferin-containing

508 medium with DZnep or vehicle (MilliQ water) were added.

509 Time-lapse imaging of PSM was performed with an inverted microscope (Olympus

510 IX81) equipped with an Olympus x10 UPlanApo objective (N.A.: 0.8) and a

511 VersArray cooled-CCD camera. 16-bit images were acquired every $5 \mathrm{~min}$ with Image-

512 Pro Plus (Media Cybernetics), with $2 \times 2$ and $4 \times 4$ binning and exposures of $100 \mathrm{~ms}$ and

$5134 \mathrm{~m} 25 \mathrm{~s}$ for DIC and chemiluminescent images, respectively. Raw imaging data were

514 processed and quantified by ImageJ (75).

515 Period and amplitude were estimated by BioDare2 (63). 
518 1. Lippi G \& Plebani M (2012) Hyperhomocysteinemia in health and disease: Where

519 we are now, and where do we go from here ?. Clin Chem Lab Med 50(12): 2075-

5202080.

521 2. Cantoni GL (1985) The role of S-adenosylhomocysteine in the biological

522 utilization of S-adenosylmethionine. Prog Clin Biol Res 198: 47-65.

523 3. Chiang PK \& Cantoni GL (1979) Perturbation of biochemical transmethylations by

524 3-deazaadenosine in vivo. Biochem Pharmacol 28(12): 1897-1902.

525 4. Hoffman DR, Marion DW, Cornatzer WE \& Duerre JA (1980) S-

526 adenosylmethionine and S-adenosylhomocystein metabolism in isolated rat liver.

527 effects of L-methionine, L-homocystein, and adenosine. J Biol Chem 255(22): 10822-

52810827.

529 5. Rana AK \& Ankri S (2016) Reviving the RNA world: An insight into the

530 appearance of RNA methyltransferases. Front Genet 7: 99.

531 6. Van Trump JE \& Miller SL (1972) Prebiotic synthesis of methionine. Science

532 178(4063): 859-860.

533 7. Waddell TG, Eilders LL, Patel BP \& Sims M (2000) Prebiotic methylation and the

534 evolution of methyl transfer reactions in living cells. Orig Life Evol Biosph 30(6):

$535 \quad 539-548$.

536 8. Fustin JM, et al (2013) RNA-methylation-dependent RNA processing controls the

537 speed of the circadian clock. Cell 155(4): 793-806. 
538 9. Guranowski A, Montgomery JA, Cantoni GL \& Chiang PK (1981) Adenosine

539 analogues as substrates and inhibitors of S-adenosylhomocysteine hydrolase.

540 Biochemistry 20(1): 110-115.

541 10. Chiang PK, Richards HH \& Cantoni GL (1977) S-adenosyl-L-homocysteine

542 hydrolase: Analogues of S-adenosyl-L-homocysteine as potential inhibitors. $\mathrm{Mol}$

543 Pharmacol 13(5): 939-947.

544 11. Tseng CK, et al (1989) Synthesis of 3-deazaneplanocin A, a powerful inhibitor of

545 S-adenosylhomocysteine hydrolase with potent and selective in vitro and in vivo

546 antiviral activities. J Med Chem 32(7): 1442-1446.

547 12. Keiser MJ, et al (2007) Relating protein pharmacology by ligand chemistry. Nat

548 Biotechnol 25(2): 197-206.

549 13. Gfeller D, Michielin O \& Zoete V (2013) Shaping the interaction landscape of

550 bioactive molecules. Bioinformatics 29(23): 3073-3079.

551 14. Yang X, et al (2003) Catalytic strategy of S-adenosyl-L-homocysteine hydrolase:

552 Transition-state stabilization and the avoidance of abortive reactions. Biochemistry

553 42(7): 1900-1909.

554 15. Kusakabe Y, et al (2015) Structural insights into the reaction mechanism of S-

555 adenosyl-L-homocysteine hydrolase. Sci Rep 5: 16641.

556 16. Brzezinski K, Dauter Z \& Jaskolski M (2012) High-resolution structures of

557 complexes of plant S-adenosyl-L-homocysteine hydrolase (lupinus luteus). Acta

558 Crystallogr D Biol Crystallogr 68(Pt 3): 218-231. 
559 17. Gomi T, et al (1990) Site-directed mutagenesis of rat liver S-

560 adenosylhomocysteinase. effect of conversion of aspartic acid 244 to glutamic acid on

561 coenzyme binding. J Biol Chem 265(27): 16102-16107.

562 18. Ault-Riche DB, Yuan CS \& Borchardt RT (1994) A single mutation at lysine 426

563 of human placental S-adenosylhomocysteine hydrolase inactivates the enzyme. $J$ Biol

564 Chem 269(50): 31472-31478.

565 19. Takata Y, et al (2002) Catalytic mechanism of S-adenosylhomocysteine

566 hydrolase. site-directed mutagenesis of asp-130, lys-185, asp-189, and asn-190. J Biol

567 Chem 277(25): 22670-22676.

568 20. Yamada T, et al (2005) Catalytic mechanism of S-adenosylhomocysteine

569 hydrolase: Roles of his 54, Asp130, Glu155, Lys185, and Asp189. Int J Biochem Cell

$570 \quad$ Biol 37(11): 2417-2435.

571 21. Sganga MW, Aksamit RR, Cantoni GL \& Bauer CE (1992) Mutational and

572 nucleotide sequence analysis of S-adenosyl-L-homocysteine hydrolase from

573 rhodobacter capsulatus. Proc Natl Acad Sci U S A 89(14): 6328-6332.

574 22. Baggs JE, et al (2009) Network features of the mammalian circadian clock. PLoS

575 Biol 7(3): e52.

23. Yoo SH, et al (2004) PERIOD2::LUCIFERASE real-time reporting of circadian

577 dynamics reveals persistent circadian oscillations in mouse peripheral tissues. Proc

$578 \quad$ Natl Acad Sci U S A 101(15): 5339-5346.

579 24. Bargiello TA, Jackson FR \& Young MW (1984) Restoration of circadian

580 behavioural rhythms by gene transfer in drosophila. Nature 312(5996): 752-754. 
bioRxiv preprint doi: https://doi.org/10.1101/653667; this version posted May 29, 2019. The copyright holder for this preprint (which was

not certified by peer review) is the author/funder, who has granted bioRxiv a license to display the preprint in perpetuity. It is made available under aCC-BY-NC-ND 4.0 International license.

581 25. Young MW, Jackson FR, Shin HS \& Bargiello TA (1985) A biological clock in

582 drosophila. Cold Spring Harb Symp Quant Biol 50: 865-875.

583 26. Hardin PE, Hall JC \& Rosbash M (1990) Feedback of the drosophila period gene

584 product on circadian cycling of its messenger RNA levels. Nature 343(6258): 536-

585540.

586

27. Kippert F, Saunders DS \& Blaxter ML (2002) Caenorhabditis elegans has a

587 circadian clock. Curr Biol 12(2): R47-9.

588 28. Saigusa T, et al (2002) Circadian behavioural rhythm in caenorhabditis elegans.

589 Curr Biol 12(2): R46-7.

29. Goya ME, Romanowski A, Caldart CS, Benard CY \& Golombek DA (2016)

591 Circadian rhythms identified in caenorhabditis elegans by in vivo long-term

592 monitoring of a bioluminescent reporter. Proc Natl Acad Sci US A 113(48): E7837-

593 E7845.

594 30. Lee S, Doxey AC, McConkey BJ \& Moffatt BA (2012) Nuclear targeting of

595 methyl-recycling enzymes in arabidopsis thaliana is mediated by specific protein

596 interactions. Mol Plant 5(1): 231-248.

597 31. Grbesa I, et al (2017) Mutations in S-adenosylhomocysteine hydrolase (AHCY)

598 affect its nucleocytoplasmic distribution and capability to interact with S-

599 adenosylhomocysteine hydrolase-like 1 protein. Eur J Cell Biol 96(6): 579-590.

600 32. Matsuo T \& Ishiura M (2011) Chlamydomonas reinhardtii as a new model system

601 for studying the molecular basis of the circadian clock. FEBS Lett 585(10): 1495-

6021502. 
bioRxiv preprint doi: https://doi.org/10.1101/653667; this version posted May 29, 2019. The copyright holder for this preprint (which was

not certified by peer review) is the author/funder, who has granted bioRxiv a license to display the preprint in perpetuity. It is made available under aCC-BY-NC-ND 4.0 International license.

603 33. van Ooijen G, et al (2013) Functional analysis of casein kinase 1 in a minimal

604 circadian system. PLoS One 8(7): e70021.

605 34. O'Neill JS, et al (2011) Circadian rhythms persist without transcription in a 606 eukaryote. Nature 469(7331): 554-558.

607 35. van Ooijen G, Dixon LE, Troein C \& Millar AJ (2011) Proteasome function is 608 required for biological timing throughout the twenty-four hour cycle. Curr Biol 609 21(10): 869-875.

610 36. Corellou F, et al (2009) Clocks in the green lineage: Comparative functional 611 analysis of the circadian architecture of the picoeukaryote ostreococcus. Plant Cell 612 21(11): 3436-3449.

613 37. Sassone-Corsi P \& Christen Y (2016) A Time for Metabolism and Hormones,

614 (Springer International Publishing : Imprint: Springer, Cham), pp 132.

615 38. Nakajima M, et al (2005) Reconstitution of circadian oscillation of cyanobacterial 616 KaiC phosphorylation in vitro. Science 308(5720): 414-415.

617 39. Johnson $\mathrm{CH}$, Zhao C, Xu Y \& Mori T (2017) Timing the day: What makes 618 bacterial clocks tick?. Nat Rev Microbiol 15(4): 232-242.

619 40. Ishiura M, et al (1998) Expression of a gene cluster kaiABC as a circadian 620 feedback process in cyanobacteria. Science 281(5382): 1519-1523.

621 41. Liu Y, et al (1995) Circadian orchestration of gene expression in cyanobacteria. 622 Genes Dev 9(12): 1469-1478. 
bioRxiv preprint doi: https://doi.org/10.1101/653667; this version posted May 29, 2019. The copyright holder for this preprint (which was

not certified by peer review) is the author/funder, who has granted bioRxiv a license to display the preprint in perpetuity. It is made available under aCC-BY-NC-ND 4.0 International license.

623

624

625

626

627

628

629 Biol Chem 281(47): 35904-35913.

640

48. Fujita H, Syono K, Machida Y \& Kawaguchi M (2008) Morphological effects of 641 sinefungin, an inhibitor of S-adenosylmethionine-dependent methyltransferases, on 642 anabaena sp. PCC 7120. Microbes Environ 23(4): 346-349.

42. Qin X, Byrne M, Xu Y, Mori T \& Johnson CH (2010) Coupling of a core posttranslational pacemaker to a slave transcription/translation feedback loop in a circadian system. PLoS Biol 8(6): e1000394.

43. Walker RD \& Duerre JA (1975) S-adenosylhomocysteine metabolism in various species. Can J Biochem 53(3): 312-319.

44. Shih PM, et al (2013) Improving the coverage of the cyanobacterial phylum using diversity-driven genome sequencing. Proc Natl Acad Sci U S A 110(3): 1053-1058.

45. HAMILL RL \& HOEHN MM (1973) A9145, a new adenine-containing antifungal antibiotic. J Antibiot (Tokyo) 26: 463.

46. Vedel M, Lawrence F, Robert-Gero M \& Lederer E (1978) The antifungal antibiotic sinefungin as a very active inhibitor of methyltransferases and of the transformation of chick embryo fibroblasts by rous sarcoma virus. Biochem Biophys Res Commun 85(1): 371-376.

47. Zheng S, et al (2006) Mutational analysis of encephalitozoon cuniculi mRNA cap (guanine-N7) methyltransferase, structure of the enzyme bound to sinefungin, and evidence that cap methyltransferase is the target of sinefungin's antifungal activity. $J$ 
644 inhibitors of bacterial DNA adenine methyltransferases. J Biomol Screen 11(5): 497-

645510.

646 50. Kageyama R, Niwa Y, Isomura A, Gonzalez A \& Harima Y (2012) Oscillatory

647 gene expression and somitogenesis. Wiley Interdiscip Rev Dev Biol 1(5): 629-641.

648 51. Kobayashi T \& Kageyama R (2014) Expression dynamics and functions of hes

649 factors in development and diseases. Curr Top Dev Biol 110: 263-283.

650 52. Rosenberg RA, Abu Haija M \& Ryan PJ (2008) Chiral-selective chemistry

651 induced by spin-polarized secondary electrons from a magnetic substrate. Phys Rev 652 Lett 101(17): 178301.

653 53. Bar-Nun A \& Hartman H (1978) Synthesis of organic compounds from carbon 654 monoxide and water by UV photolysis. Orig Life 9(2): 93-101.

655 54. Sarker PK, Takahashi J, Obayashi Y, Kaneko T \& Kobayashi K (2013) Photo656 alteration of hydantoins against UV light and its relevance to prebiotic chemistry. 657 Advances in Space Research 51(12): 2235-2240.

658 55. Powner MW, Gerland B \& Sutherland JD (2009) Synthesis of activated 659 pyrimidine ribonucleotides in prebiotically plausible conditions. Nature 459(7244): $660239-242$.

661 56. O'Neill JS \& Reddy AB (2011) Circadian clocks in human red blood cells. Nature 662 469(7331): 498-503. 
663 57. Edgar RS, et al (2012) Peroxiredoxins are conserved markers of circadian

664 rhythms. Nature 485(7399): 459-464.

665 58. Feeney KA, et al (2016) Daily magnesium fluxes regulate cellular timekeeping 666 and energy balance. Nature 532(7599): 375-379.

667 59. Tan J, et al (2007) Pharmacologic disruption of polycomb-repressive complex 2668 mediated gene repression selectively induces apoptosis in cancer cells. Genes Dev 669 21(9): 1050-1063.

670 60. Miranda TB, et al (2009) DZNep is a global histone methylation inhibitor that 671 reactivates developmental genes not silenced by DNA methylation. Mol Cancer Ther 672 8(6): 1579-1588.

673 61. Zeybel M, et al (2017) A proof-of-concept for epigenetic therapy of tissue 674 fibrosis: Inhibition of liver fibrosis progression by 3-deazaneplanocin A. Mol Ther $675 \quad 25(1): 218-231$.

676 62. Nitanda Y, et al (2014) 3'-UTR-dependent regulation of mRNA turnover is 677 critical for differential distribution patterns of cyclic gene mRNAs. Febs $J$ 281(1): $678 \quad 146-156$.

679 63. Zielinski T, Moore AM, Troup E, Halliday KJ \& Millar AJ (2014) Strengths and 680 limitations of period estimation methods for circadian data. PLoS One 9(5): e96462.

681 64. Vallone D, Gondi SB, Whitmore D \& Foulkes NS (2004) E-box function in a 682 period gene repressed by light. Proc Natl Acad Sci U S A 101(12): 4106-4111. 
bioRxiv preprint doi: https://doi.org/10.1101/653667; this version posted May 29, 2019. The copyright holder for this preprint (which was

not certified by peer review) is the author/funder, who has granted bioRxiv a license to display the preprint in perpetuity. It is made available under aCC-BY-NC-ND 4.0 International license.

683 65. Lamba P, Foley LE \& Emery P (2018) Neural network interactions modulate

684 CRY-dependent photoresponses in drosophila. J Neurosci 38(27): 6161-6171.

685 66. Chen C, Xu M, Anantaprakorn Y, Rosing M \& Stanewsky R (2018) Nocte is

686 required for integrating light and temperature inputs in circadian clock neurons of

687 drosophila. Curr Biol 28(10): 1595-1605.e3.

688 67. Mello CC, Kramer JM, Stinchcomb D \& Ambros V (1991) Efficient gene transfer

689 in C.elegans: Extrachromosomal maintenance and integration of transforming

690 sequences. Embo J 10(12): 3959-3970.

691 68. Hansen LL \& van Ooijen G (2016) Rapid analysis of circadian phenotypes in 692 arabidopsis protoplasts transfected with a luminescent clock reporter. $J$ Vis Exp (115).

693 doi(115): 10.3791/54586.

694 69. Hwang S, Kawazoe R \& Herrin DL (1996) Transcription of tufA and other

695 chloroplast-encoded genes is controlled by a circadian clock in chlamydomonas. Proc

696 Natl Acad Sci U S A 93(3): 996-1000.

697 70. Matsuo T, et al (2008) A systematic forward genetic analysis identified

698 components of the chlamydomonas circadian system. Genes Dev 22(7): 918-930.

699 71. Okamoto K, Onai K, Ezaki N, Ofuchi T \& Ishiura M (2005) An automated

700 apparatus for the real-time monitoring of bioluminescence in plants. Anal Biochem

$701 \quad 340(2): 187-192$.

702 72. Xu Y, Mori T \& Johnson CH (2003) Cyanobacterial circadian clockwork: Roles

703 of KaiA, KaiB and the kaiBC promoter in regulating KaiC. Embo J 22(9): 2117-2126. 
bioRxiv preprint doi: https://doi.org/10.1101/653667; this version posted May 29, 2019. The copyright holder for this preprint (which was

not certified by peer review) is the author/funder, who has granted bioRxiv a license to display the preprint in perpetuity. It is made available under aCC-BY-NC-ND 4.0 International license.

704 73. Kondo T, et al (1993) Circadian rhythms in prokaryotes: Luciferase as a reporter

705 of circadian gene expression in cyanobacteria. Proc Natl Acad Sci U S A 90(12):

$706 \quad 5672-5676$.

707 74. Bustos SA \& Golden SS (1991) Expression of the psbDII gene in synechococcus

708 sp. strain PCC 7942 requires sequences downstream of the transcription start site. $J$

709 Bacteriol 173(23): 7525-7533.

710 75. Shimojo H, Harima Y \& Kageyama R (2014) Visualization of notch signaling

711 oscillation in cells and tissues. Methods Mol Biol 1187: 169-179.

712

713 
Fig. 1: Adenosylhomocysteinase is a highly conserved protein. (a) Structural

717 superposition of AHCY from the 9 organisms investigated here, using human (1LI4), 718 mouse (5AXA) or lupin (3OND) crystal structures as templates. The blue loop is 719 specific to plants and green algae; DZnep is shown in yellow, $\mathrm{NAD}^{+}$in grey. See also 720 Movie S1. (b) Docking simulation of human AHCY with DZnep, based on the 1LI4 721 crystal structure of human AHCY complexed with Neplanocin A, an analogue of 722 DZnep. The amino acids involved in DZnep binding are indicated with their position. 723 See Fig. S2 for docking simulations of DZnep to AHCY from other organisms. Red 724 and green arrows are hydrogen bonds, yellow spheres are hydrophobic effects. The estimated free energy of binding for depicted DZnep docking conformation was -9.87 $\mathrm{kcal} / \mathrm{mol}$. (c) Discontinuous alignment of amino acids contributing to the binding of DZnep, using the human sequence as a reference and with sequence identities shown on the right. When amino acids are identical to human, a dot is shown in the

729 alignment. The sequence logo on top is a graphical representation of the conservation 730 of amino acids, with the consensus symbols below $(*=$ fully conserved residue, $:=$ 731 conservation of strongly similar properties, . = conservation of weakly similar 732 properties). The positions of selected conserved amino acids are given for the human 733 sequence. (d) Molecular docking simulations of AHCY with adenosine (top) or 734 DZnep (below) showing comparable binding free energies in all organisms. Colors represent full sequence identities, relative to human.

737 Fig. 2: The link between the methyl cycle and the clock is conserved in 738 vertebrates. (a) Left panel shows mean luminescence $+/-$ SEM of human U-2 OS 
739 cells stably transfected by a Bmall-luc reporter and treated with increasing

740 concentrations of DZnep. Middle panel shows mean $+/$ - SEM of period, $n=3$. Right

741 panel shows mean +/- SEM of amplitude, $\mathrm{n}=3$ dishes. Same analyses were

742 performed with PER2::LUC mouse embryonic fibroblasts, $\mathrm{n}=3$ dishes (b), and PAC-

7432 zebrafish cells stably transfected with a Perlb-luciferase reporter, $\mathrm{n}=4$ dishes,

744 mean +/- SEM (c). These experiments were independently reproduced at least three

745 times. All bar graphs analyzed by One-Way ANOVA followed by Bonferroni post-

746 hoc test; all indicated comparisons at least $p<0.05$.

Fig. 3: The link between the methyl cycle and the clock is conserved in

749 invertebrates. (a) Real-time luminescence from halteres in culture dissected from 750 ptim-TIM-LUC male flies. Luminescence traces show mean $+/$ - SEM of $\mathrm{n}=8$

751 halteres, with only the upper segment of the error bars shown for clarity. Middle and 752 right panel shows mean $+/$ - SEM of period and amplitude, respectively, for $\mathrm{n}=$ at 753 least 8 halteres for each treatment group. All bar graphs analyzed by One-Way 754 ANOVA followed by Bonferroni post-hoc test; all indicated comparisons at least $p<$ 755 0.05. (b) Locomotor activity counts from detrended luminescence measurements of 756 freely moving Caenorhabditis elegans populations of $\sim 100$ nematodes treated with 757 vehicle or $100 \mu \mathrm{M}$ DZnep, showing mean $+/$ - SEM of $n=10$ populations. In the 758 middle, population mean $+/$ - SEM of period (left) and amplitude (right) compared by 759 Student $t$-test ; **, $p<0.01 ; \mathrm{n}=10$ populations treated with vehicle and $\mathrm{n}=6$ treated 760 with DZnep. The right panel shows mean $+/-$ SEM of period obtained from single 761 nematodes in isolation, $\mathrm{n}=21$ controls and 22 for DZnep, analyzed by Student $t$-test, $762 \quad * *, p<0.01$. 
764 Fig. 4: The link between the methyl cycle and the clock is conserved in plants. (a)

765 Left panel shows mean luminescence +/- SEM of Arabidopsis thaliana protoplasts

766 bearing a CCA1pro:LUC reporter construct, $\mathrm{n}=8$ wells per treatment, treated with 767 different concentration of DZnep. For comparison between different runs, traces were 768 aligned in relation to the first peak. Middle panel shows mean +/- SEM of amplitude, $769 \mathrm{n}=8$. Right panel shows mean $+/$ - SEM of amplitude, $\mathrm{n}=8$. (b) Same as (a) but with 770 Ostreococcus tauri cells carrying a CCA1-LUC reporter, $\mathrm{n}=8$ wells. Middle panel 771 shows mean $+/-$ SEM of period, $\mathrm{n}=8$ wells. No significance was observed between 77210,20 and $30 \mu \mathrm{M}$, but the significance compared to $0 \mu \mathrm{M}$ became stronger, i.e. $p<$ $7730.05, p<0.001, p<0.0001$, respectively, indicating dose-dependent effects. Right 774 panel shows mean $+/-$ SEM of amplitude, $\mathrm{n}=8$ wells. (c) Same as (b) but with 775 Chlamydomonas reinhardtii $\mathrm{CBR}$ strain cultures stably-transfected with a tufA 776 promoter-lucCP reporter, $\mathrm{n}=5$ wells. Middle panel shows mean $+/$ - SEM of period, $\mathrm{n}$ $777=5$ wells. Right panel shows mean $+/-$ SEM of amplitude, $\mathrm{n}=5$ wells. All bar graphs analyzed by One-Way ANOVA followed by Bonferroni post-hoc test; all indicated comparisons at least $p<0.05$.

Fig. 5: The link between the methyl cycle and the clock is conserved in

cyanobacteria. (a) Left panel shows mean luminescence +/- SEM of Synechococcus

783 PCC 7942 kaiBCp::luxAB knock-in strain treated with different concentrations of DZnep, $\mathrm{n}=3$, with only the upper section of the error bars shown for clarity. Middle panel shows mean period +/- SEM, $\mathrm{n}=3$. Right panel shows mean amplitude $+/-$

786 SEM, $\mathrm{n}=3$. (b) Same as (a) but using Synechococcus PCC 7942 psbAIp::luxAB 787 knock-in strain. Data for (a) and (b) were analyzed together with data from Fig. S3, 788 showing additional concentrations of DZnep. (c) and (d) are the same as (a) and (b), 
respectively, but with different Sinefungin concentrations as indicated over the graphs.

790 In addition, bar charts in (c) and (d) show data obtained with higher concentrations of

791 Sinefungin presented in Fig. S3. See also Fig. S4 for a comparison with EGX ${ }^{\mathrm{I}}$. All bar 792 graphs analyzed by One-Way ANOVA followed by Bonferroni post-hoc test; all 793 indicated comparisons at least $p<0.05$.

Fig. 6: The somite segmentation clock is sensitive to methyl cycle inhibition. (a)

796 Representative detrended luminescence measurements from one cultured embryo per 797 treatment as indicated in the legend on the right. (b) Mean period $+/-$ SEM of $n=3$ 798 embryos per treatment, with $*=p<0.05$. (c) Mean amplitude $+/-$ SEM of $\mathrm{n}=3$ 799 embryos per treatment, with $* *=p<0.01$. (d) Montage of luminescence time-lapse 800 micrographs of one representative embryo for each treatment. One picture every 20 801 min is shown, starting from top left, each row corresponding to one $H e s 7$ expression 802 cycle in the embryo treated with $0 \mu \mathrm{M}$ DZnep, as shown in (a). See also Movies S2 803 and S3. (e) Anatomic localization of the presomitic mesoderm in embryo and 804 schematic representation of the pictures from (d). The yellow square defined the area 805 from which luminescence was measured in (a). 

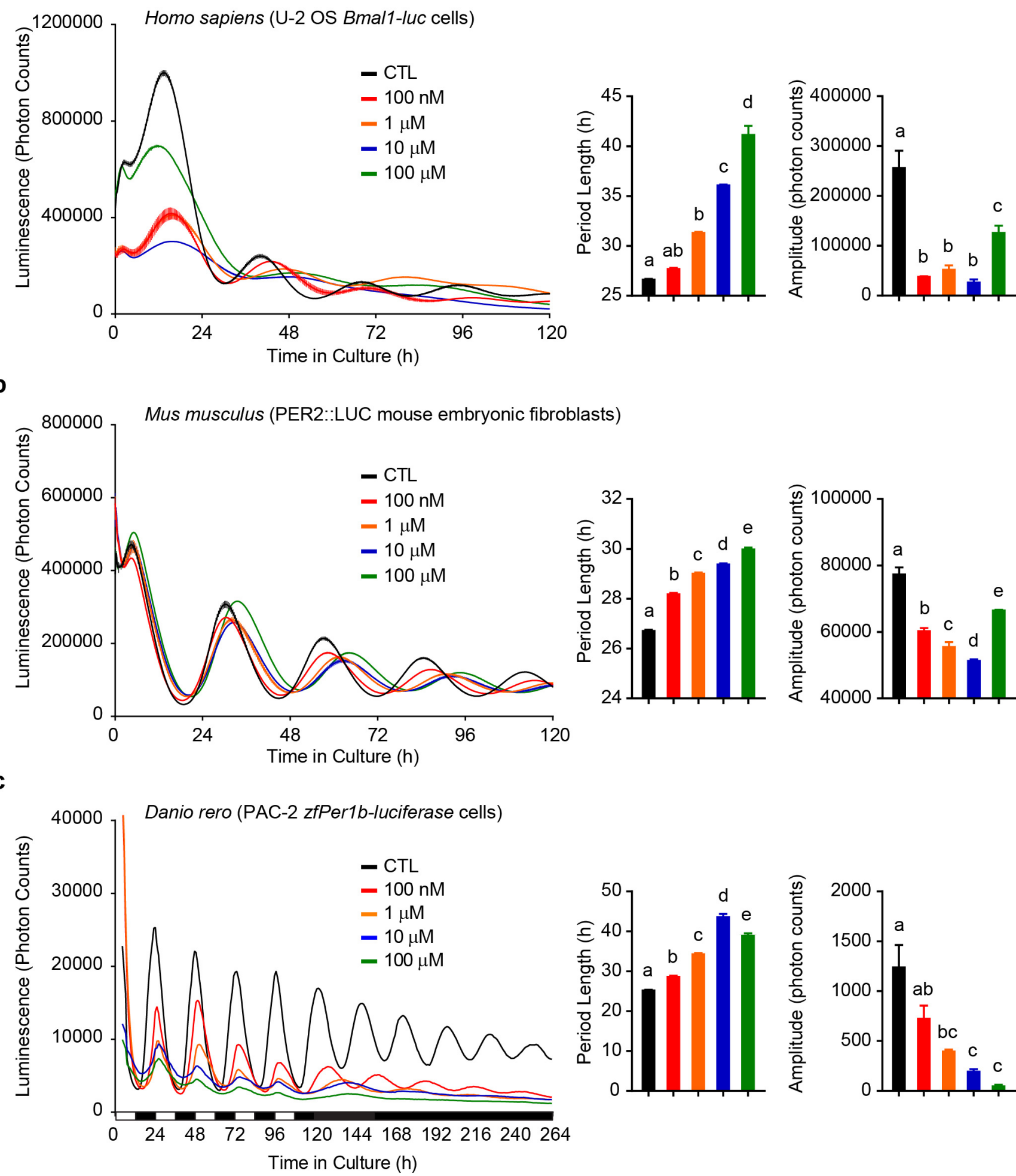

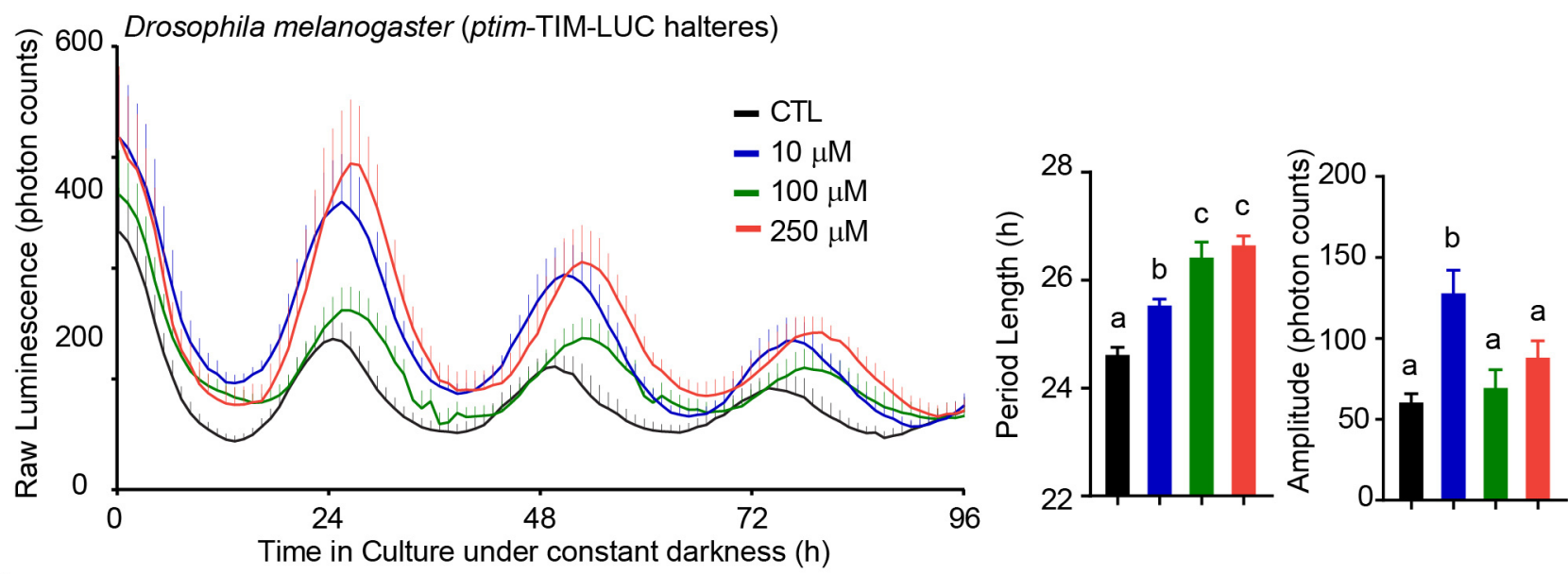

b
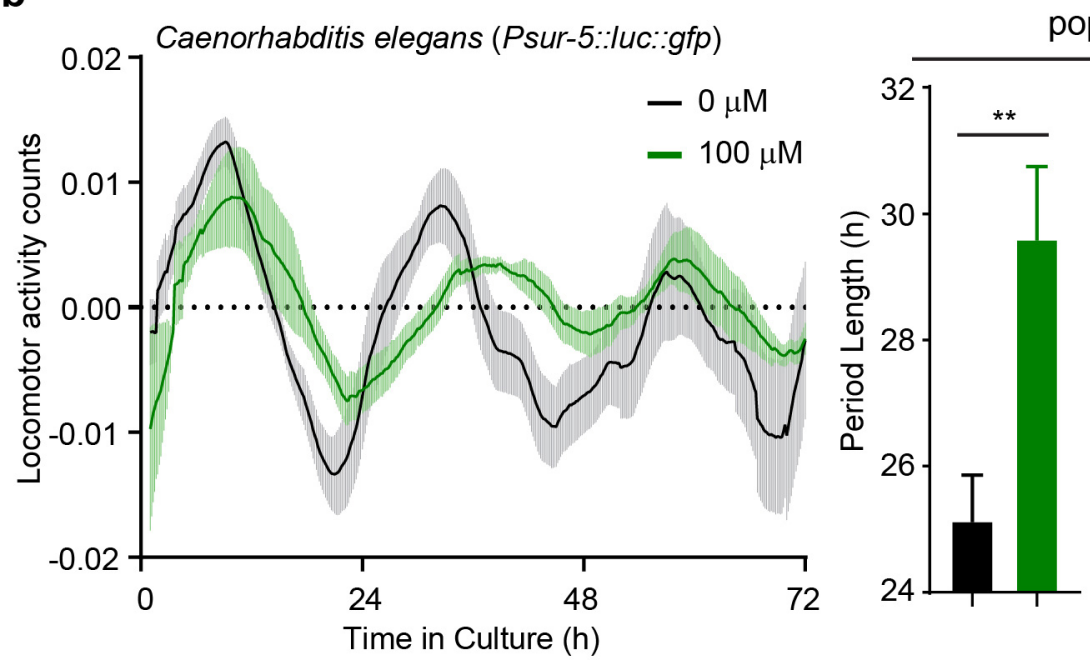

populations

single worms
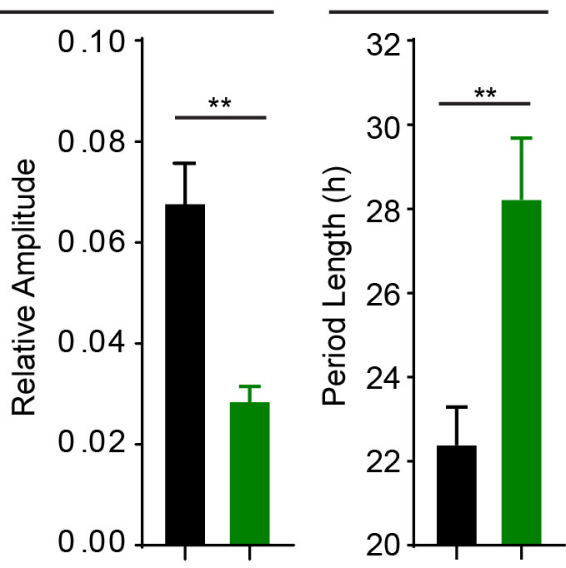

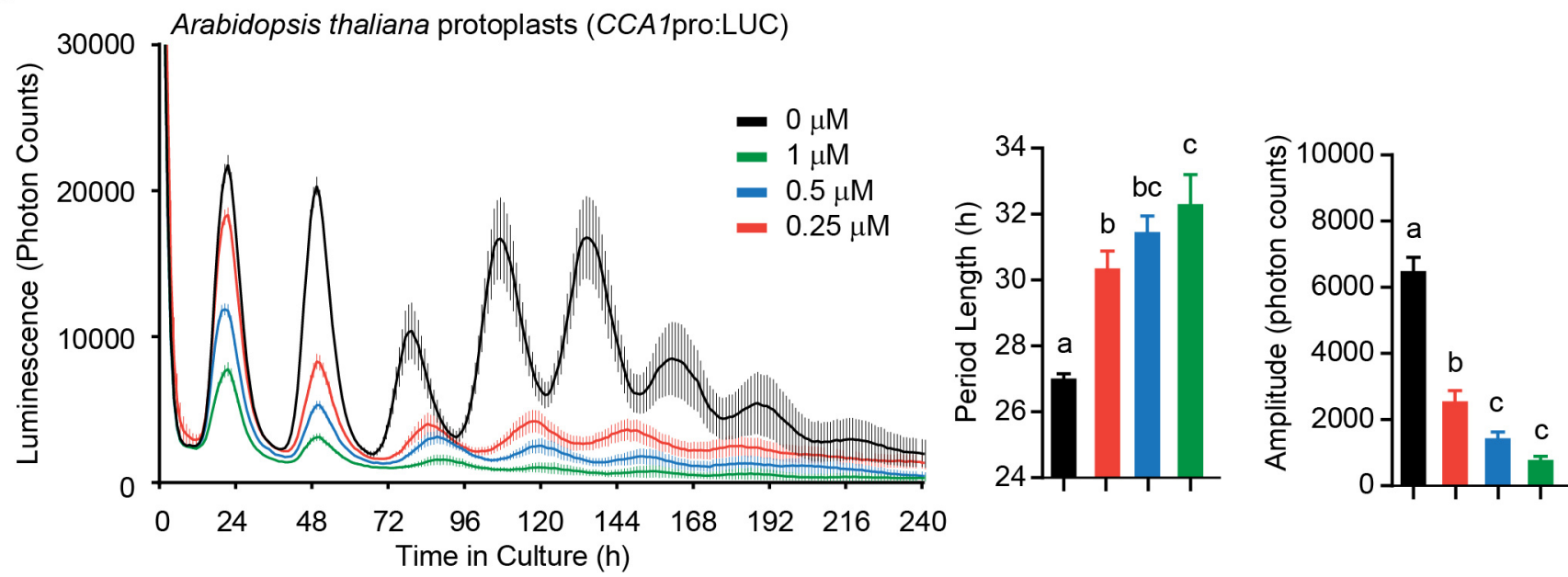

b

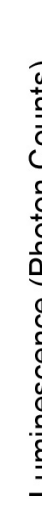

300000 Ostreococcus tauri (CCA-luc)
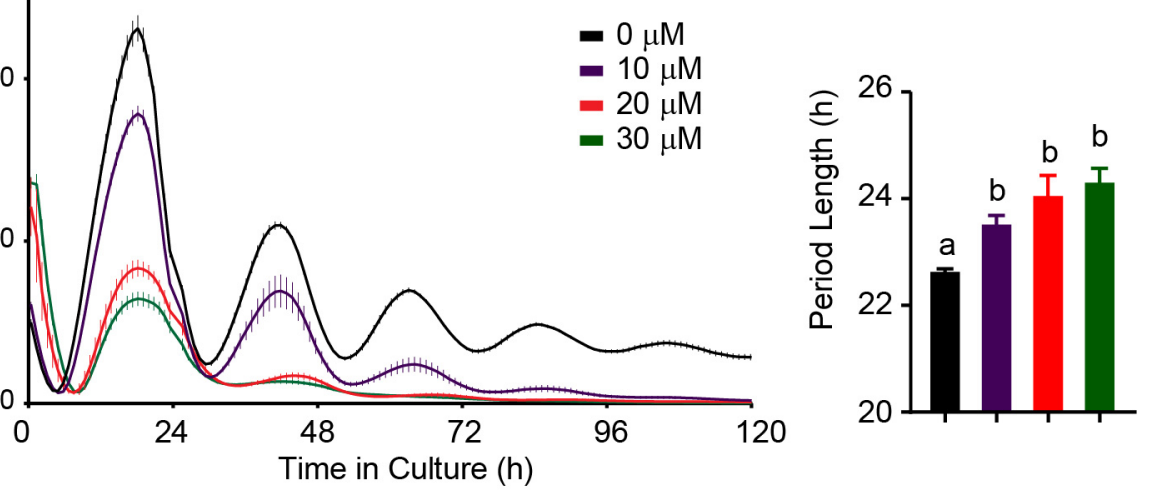

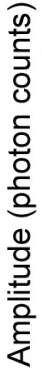

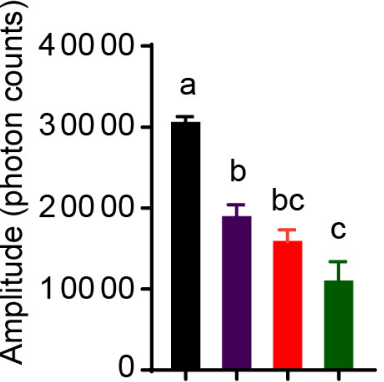

C

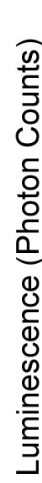

30000 Chlamydomonas reinhardtii CBR strain (tufA promoter-lucCP)
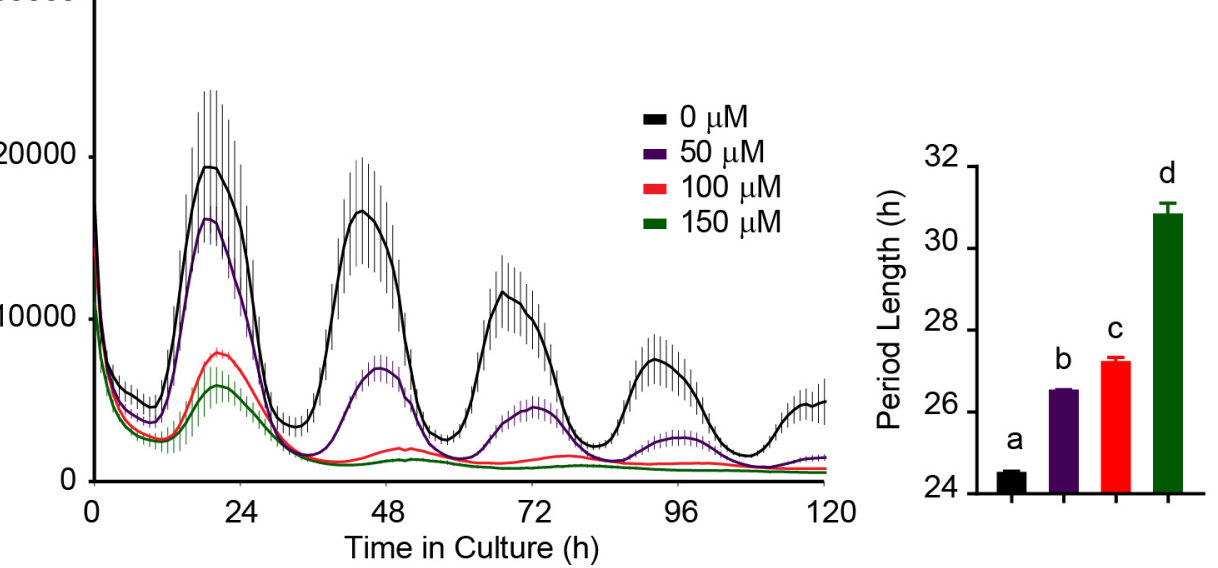

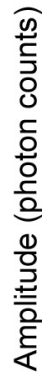

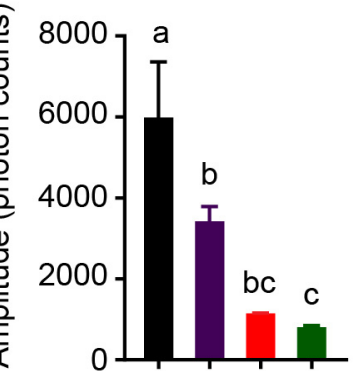




\section{a Synechococcus PCC 7942 kaiBCp::IuxAB}
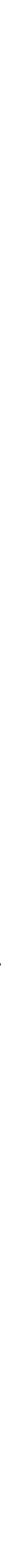

d
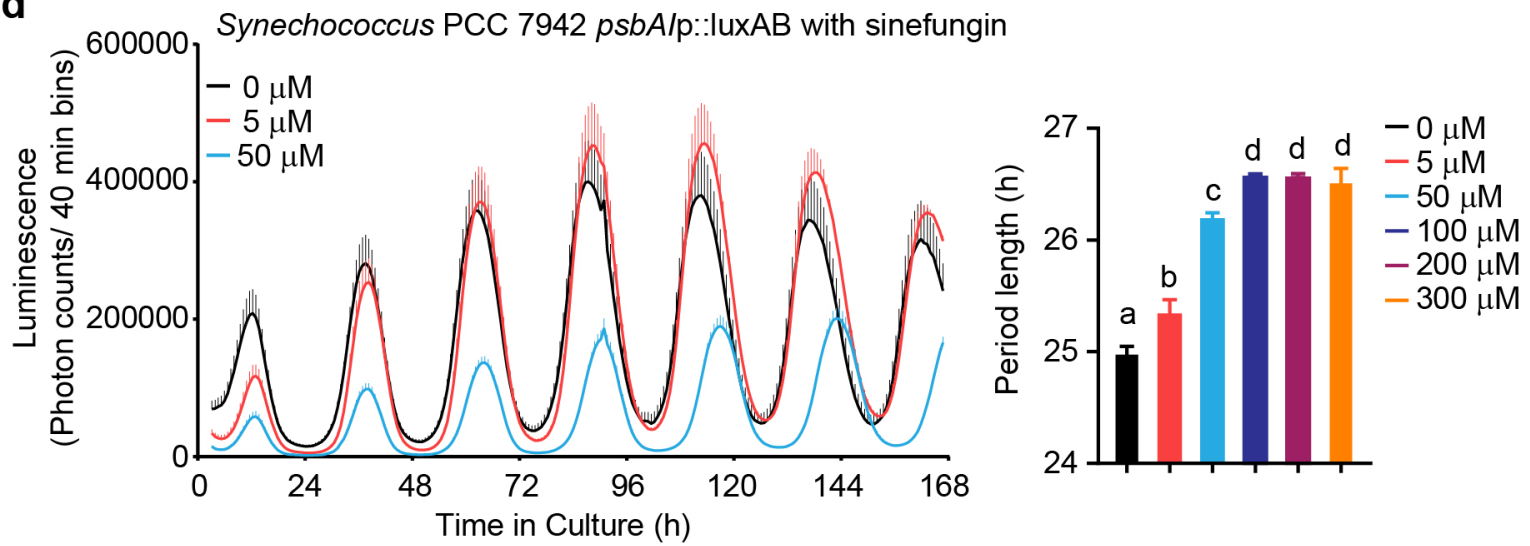
